MATEC Web of Conferences 24,07001 (2015)

DOI: $10.1051 /$ matecconf/ 20152407001

C Owned by the authors, published by EDP Sciences, 2015

\title{
Quantification of human-structure interaction
}

\author{
Colin Caprani ${ }^{1, a}$, Junnan $\mathrm{Qu}^{1}$, Stana Zivanovic ${ }^{2}$, Neil Evans ${ }^{2}$, and Ehsan Ahmadi ${ }^{1}$ \\ ${ }^{1}$ Monash University, Department of Civil Engineering, Melbourne, Australia \\ ${ }^{2}$ Warwick University, School of Engineering, Coventry, United Kingdom
}

\begin{abstract}
In lightweight structural systems there is increasing evidence that the presence of humans influences the dynamics characteristics of the system. In the past, most effort on determining the footfall-induced vertical force to the walking surface has been conducted using rigid or non-flexible surfaces such as treadmills. However, should the walking surface be vibrating, the characteristics of human walking could change to maximize comfort. This interaction between the structure and human may account for the discrepancy between the levels of vibration predicted by theory and those observed in practice. Indeed, many design rules can be seen to be conservative, perhaps partly because knowledge of this human-structure interaction is limited. This work aims to address this problem by quantifying the magnitude of human-structure interaction through a comprehensive experimental programme. Novel experimental techniques are used to measure the human-imparted force on the walking surface. Both rigid and flexible (vibrating) surfaces are used, and we measure the imparted vibration response on a lively footbridge (the Warwick Bridge) which acts as the flexible surface. A range of test subjects is considered, walking at a range of pacing frequencies. Comparison is made between a notional vibration response from the footfall force imparted to the rigid surface and the actual vibration response caused by the footfall force imparted to the flexible surface. Key aspects of the experimental regime are also explained. Finally, some comparisons are made using footfall force models from the literature. It is concluded that human-structure interaction is a key phenomenon that should be taken into account in the design and assessment of vibration-sensitive structures.
\end{abstract}

\section{Introduction}

Many newly built structures have difficulties satisfying vibration serviceability criteria when occupied and dynamically excited by humans, such as footbridges, grandstands, staircases and open-plan floors [1]. The problems have been caused typically by human occupants performing normal daily activities such as walking, running, jumping, bouncing/bobbing and dancing. Vibration beyond the normal range will influence pedestrian comfort while occupying the structure. As a classic example, the London Millennium Bridge in 2000 was temporarily closed following excessive pedestrianinduced vibrations during its inauguration [2].

There have been numerous attempts to provide reliable and practical descriptions of pedestrian-induced forces by measuring the contact forces, or ground reaction forces (GRFs), between the ground and pedestrian [3], [4]. Present state-of-the-art GRF measurement facilities typically comprise equipment for direct force measurements, such as a force plate and an instrumented treadmill usually mounted on rigid laboratory floors. However, a change in GRFs could occur due to the structure flexibility. For example, Ohlsson [5] found that the force measured on a flexible timber floor is different from that measured on a rigid base, and Pavic et al. [6] pointed out that the force obtained on a flexible concrete beam was two times lower than that on a force plate. It has been demonstrated recently that using GRF measurements to directly calculate structural response under walking humans is justifiable in the case of civil engineering structures which do not vibrate perceptibly [7].

The human body is a very sensitive vibration receiver characterized by an innate ability to adapt quickly to almost any type and level of vibration which normally occurs in nature [8]. Moreover, this effective selfadapting mechanism triggers pedestrians to change walking behaviour, and hence GRFs [9]. This leads to force patterns different from the corresponding rigidground measurements [10]. This interdependency between the movement of pedestrians and the vibration response of the structure, whose motion is perceived, is usually referred to as human-structure interaction (HSI). However, as noted by [11], human-structure interaction is only one aspect of a more complex phenomenon influencing human-induced forces. Indeed, HSI is associated not only with the forces that excite the structure, but also with the corresponding influence of humans on the dynamic properties of the structure they occupy (i.e. modal mass, damping and stiffness) [12], [13].

The present work aims to experimentally capture the difference between vibration response caused by GRFs

\footnotetext{
a Corresponding author: colin.caprani@monash.edu
} 
measured from rigid and flexible surfaces. It also aims to establish the inaccuracies in modelling vibration response, and the response predictions of the current state-of-the-art. The initial results of the measured and analytical modelling are given.

\section{Experimental programme}

Figure 1 schematically illustrates an overview of the whole experimental programme to investigate HSI phenomenon. Two types of test are carried out in order to quantify HSI. First, GRFs on a rigid surface (RS) are measured. Then, GRFs are measured from walking on a vibrating bridge surface (BS) and the vibration response concurrently measured. Hypothetical vibration responses are also inferred from the RS-measured GRFs using a modal model of the bridge. The comparison of the vibration response obtained from GRFs on RS and BS can reflect the influence of the vibrating bridge surface on the walking process. Also, comparing measure vibration responses from real bridge to those recommended in the literature can show the accuracy of the state-of-the art. Going a step farther, comparing vibration response from GRFs on BS using the analytical model to those of measured from the real bridge can result in a good estimation of bridge frequency and damping change due to the pedestrian. In fact, in this paper, the influence of the pedestrian on the bridge frequency and damping are considered.

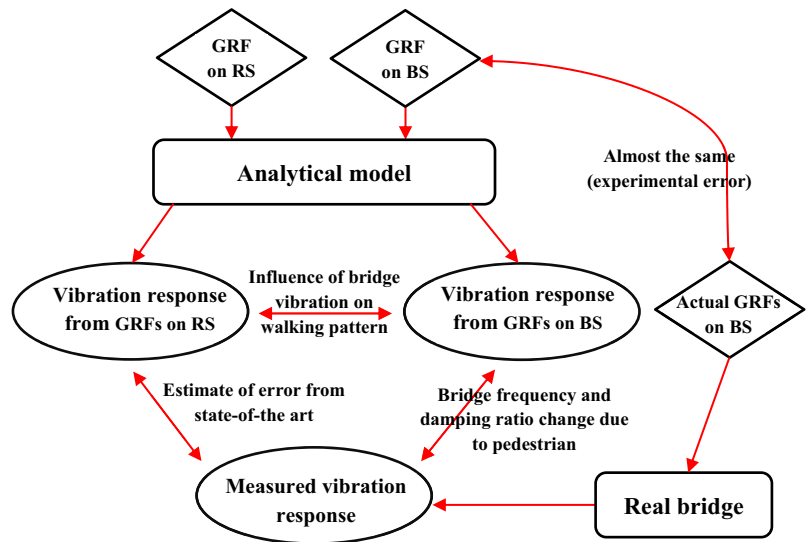

Figure 1 A schematic overview of the experimental programme (see text for definition of acronyms).

\subsection{Bridge properties, free decay vibration, and trials}

All the tests were carried out on the steel-concrete composite laboratory footbridge in Warwick University, UK. The bridge is a unique laboratory structure that exhibits excessive vibration in the vertical direction making it an ideal facility for studying HSI (Figure 2). The span length of the bridge is adjustable and the tested span is $16.2 \mathrm{~m}$ long and $2 \mathrm{~m}$ wide. Mass per unit length of the bridge is $760 \mathrm{~kg} / \mathrm{m}$. Bridge damping and frequency are determined using free vibration decay as will be described later.

To test HSI at resonance, the pacing frequency $\left(f_{p}\right)$ is set to 145 beats/min $(2.4 \mathrm{~Hz})$, which is very close to the bridge natural frequency $\left(f_{b}\right)$. Trials were also carried out at 73 beats $/ \min (1.2 \mathrm{~Hz})$, which is near to half pacing frequency (second walking force harmonic in resonance). These tests can investigate the influence of human force harmonics on bridge vibration response. To investiagte HSI when walking does not excite bridge resonance, a pacing frequency of $127 \mathrm{beats} / \mathrm{min}(2.1 \mathrm{~Hz})$ is tested as well.

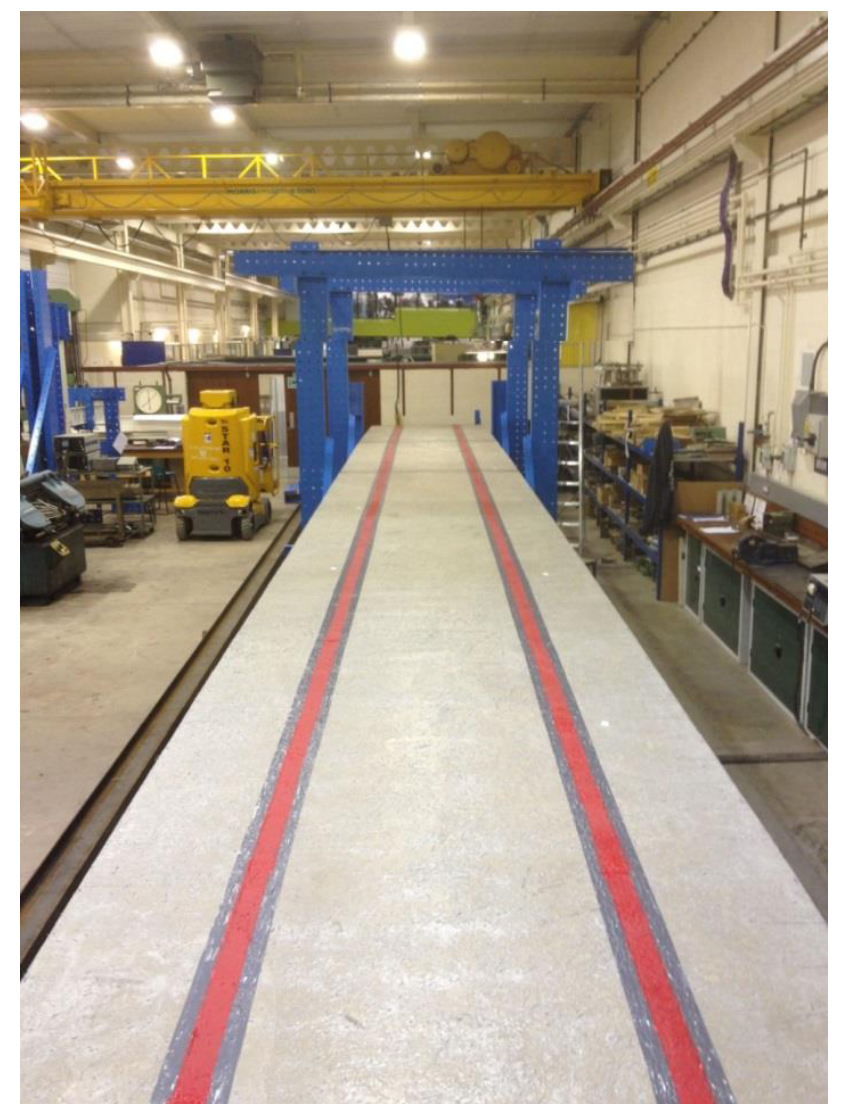

Figure 2 The Warwick bridge.

Three test subjects who participated in the experimental tests are considered in this paper. They have weights of $646 \mathrm{~N}, 793 \mathrm{~N}, 968 \mathrm{~N}$ and are denoted as TS1, TS2, and TS3, respectively. The walking trials were repeated for all three pacing frequencies mentioned above. Each pedestrian also performed a trial at a personally preferred walking frequency, termed 'natural' walking pacing frequency. In the experiments, each pacing frequency for each test subject had at least five trials because data acquisition during some trials was problematic. Therefore, the useable trials are four for each pacing frequency and used as the sample data.

In each trial, the test subject walked a circuit including the rigid surface (RS) and bridge surface (BS). On both the two surfaces, the walking lengths were the same. After a sound signal, the pedestrian started to walk. A metronome was used during the walking so that pedestrian could at the desired pacing frequency. The means of collecting GRFs will be described later. The mid-span acceleration response of the bridge for a test subject with pacing frequency of 145 beats/min $(2.4 \mathrm{~Hz})$ is shown in Figure 3. 


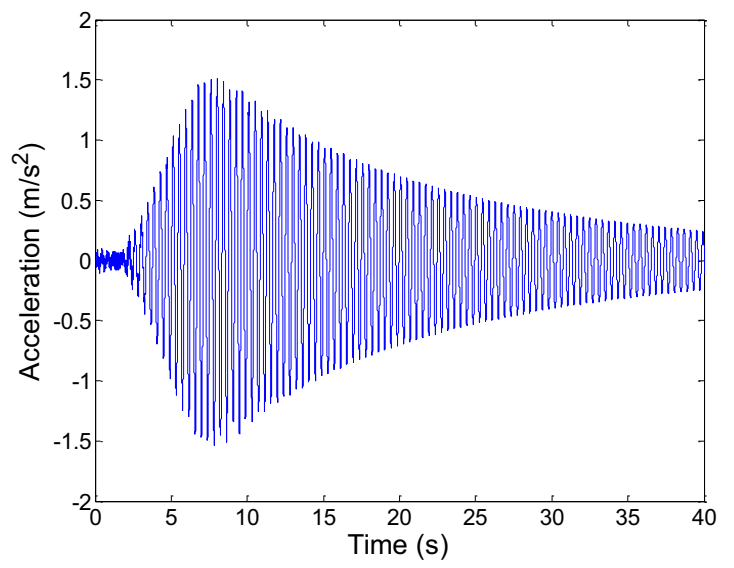

Figure 3 Acceleration response at mid-span for one test subject.

Noise in the measured bridge acceleration data was removed by low-pass Butterworth filter, in which the cutoff frequency is $20 \mathrm{~Hz}$. The corresponding filtered power spectrum density (PSD) of the acceleration signal in Figure 4 reveals that most of the response energy is concentrated at the first forcing harmonic and at the first vibration mode. So, only first harmonic of the force and first vibration mode of the bridge is considered in the subsequent analytical modelling. As expected, the bridge was extremely lively in this test, with peak acceleration level almost reaching $1.5 \mathrm{~m} / \mathrm{s}^{2}$. The first natural frequency is about $2.38 \mathrm{~Hz}$.

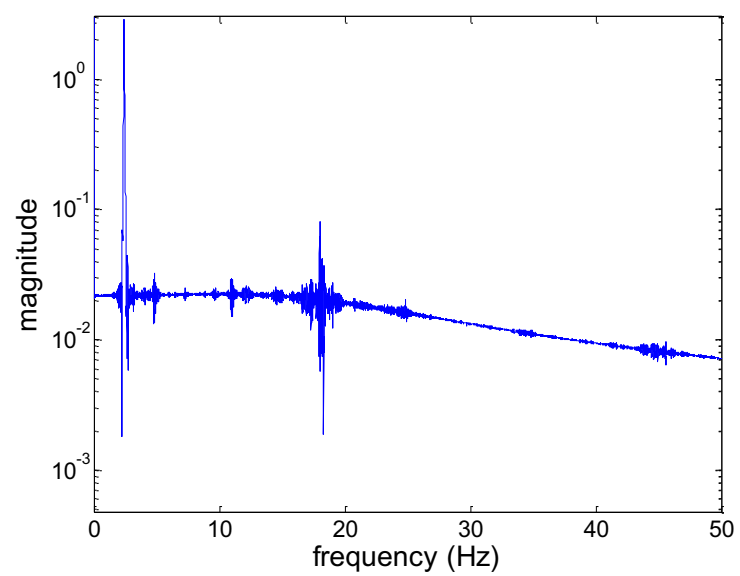

Figure 4 Filtered PSD of the acceleration response at mid-span

To determine the damping $(\xi)$ of the bridge from free decay vibration, an exponential decay curve is leastsquares fit to a moving window of 5 peaks. (Figure 5). As is common, it is seen that the damping ratio increases with increase in the vibration amplitude. This is because of more sources of energy dissipation at higher vibration amplitudes. Nevertheless, the maximum achieved value of the damping ratio is still quite low, which ensures the lively behaviour of the structure necessary for the interaction studies. Finally, a curve is fit (as show in Figure 5) to approximate the relationship between damping and amplitude.

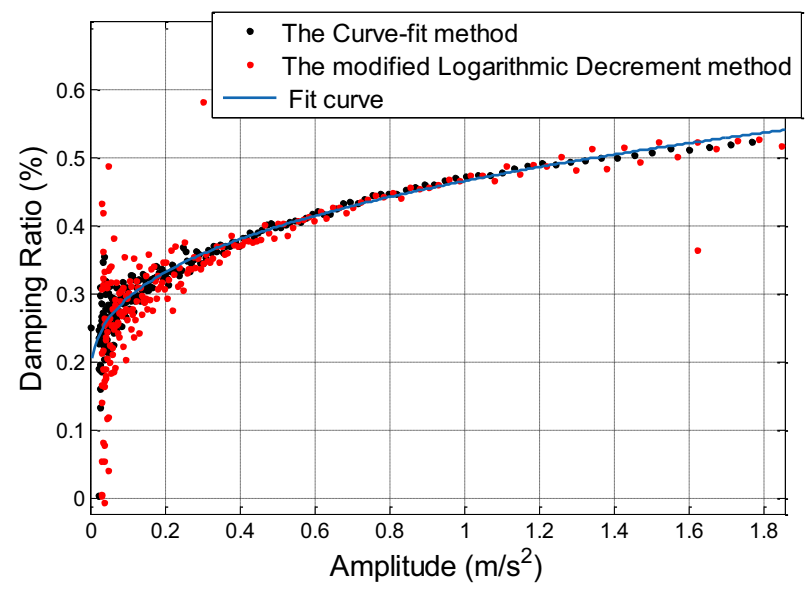

Figure 5 Amplitude dependency of bridge damping ratio in the fundamental mode of vibration.

The natural frequency was also extracted from the free vibration decay response by analyzing frequency of each cycle during a full vibration signal. Figure 6 shows that the natural frequency decreases with increasing vibration amplitude, and again an approximating fitted curve is shown.

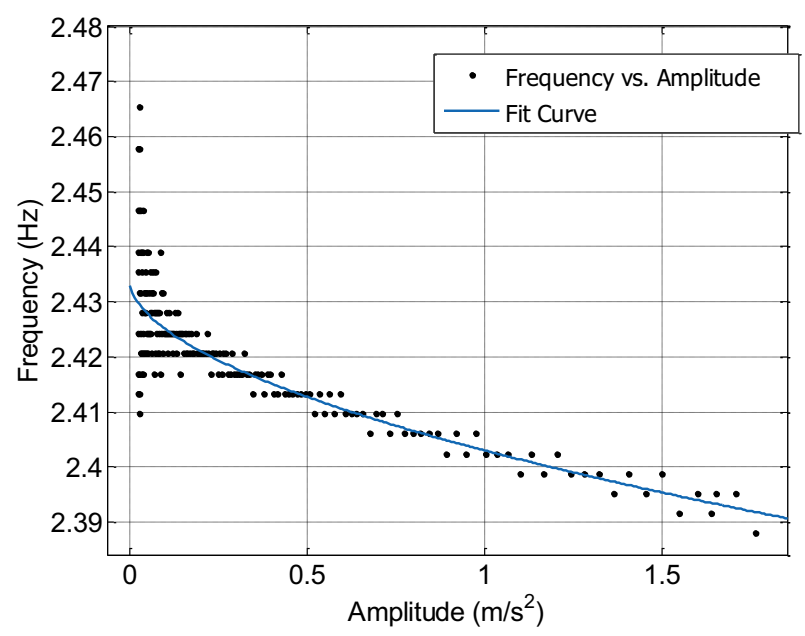

Figure 6 Amplitude dependency of natural frequency.

\subsection{Force data acquisition and processing}

To measure GRFs, Tekscan in-shoe foot pressure sensors are used. These provide dynamic pressure, force and timing information for foot function and gait analysis. An example of the resulting force signal as well as pressure distribution on each foot obtained from the TekScan FScan system is shown in Figure 7.

The TekScan F-Scan software supports five methods for calibrating sensors: point calibration, step calibration, walk calibration, frame calibration and two-point calibration. All of these methods were trialled for accuracy using a force plate before the full experiment trials were conducted. Of most interest, step calibration and walk calibration use the known subject's weight to adjust the calibration factor. Following the trials, a walk calibration was found to give higher accuracy compared to step calibration using the same factors. 


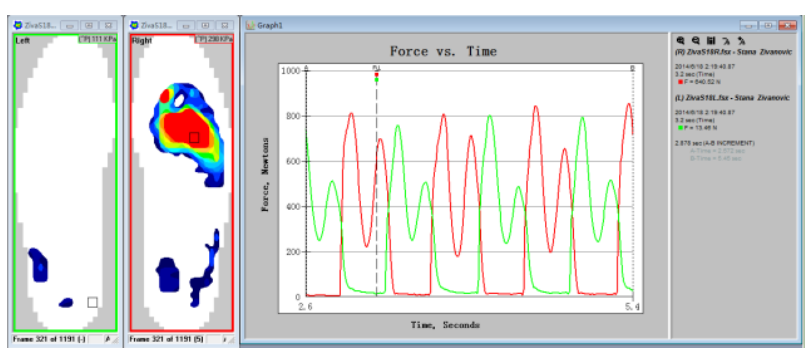

Figure 7 An example of force signal plus pressure distribution on each foot obtained from F-Scan.

There are some potential sources of error when using TekScan. Most notably, due to degradation of the sensor, drift of the sensor output can occur over time. To account for this, calibration of the sensor is carried out for each trial using the known subject weight and rigid surface walk force-time history. Thus, each trial has its own calibration factor. Additionally, the sensors can deteriorate so that rows or columns of the 'sensels' no longer export forces, and in these cases the walk calibration approach may not be succesful.

Figure 8 shows a sample of the calibration factor (indicated by "saturation pressure") for TS1 for pacing frequency of 145 beats $/ \mathrm{min}$. It can be seen that the accuracy of trials is reasonably reliable because the saturation pressures of the trials carried out of a period of about 4-5 hours are similar.

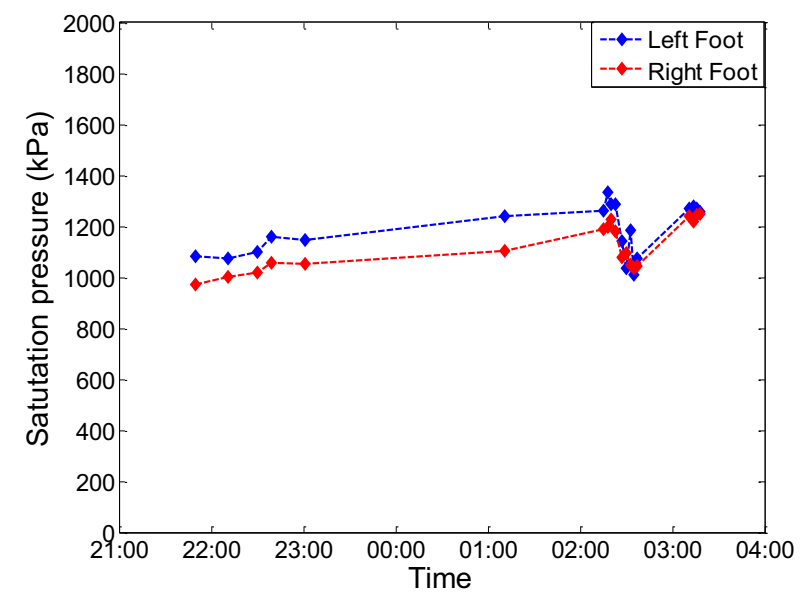

Figure 8 Saturation pressure vs. time (hours) for TS1 and pacing frequency of $145 \mathrm{beat} / \mathrm{min}$.

Finally, it is also necessary to zero the sensor output. Indeed, when one foot is supporting the body weight during walking, the force of the foot should be zero. However, because the foot sensors are pre-tensioned to the sole of the foot by shoe-lacing, the output of sensors isn't be zero when foot isn't touching the ground. Hence it is necessary to zero the force output for each trial during a swing phase.

\section{Analytical model}

The modal analysis model used is shown in Figure 9. The pedestrian is modelled as a moving force with constant velocity and the bridge is modelled as a simply supported beam in the modal space considering only the first mode of the vibration. The exact average velocity of the test subjects during each trial is used and found by dividing the bridge length by test subject crossing time. The moving force could be either a harmonic force given in the literature or force signal measured during the experiment on the RS or BS:

$$
F(t)=w_{P} \sum_{k=0}^{n} \eta_{k} \cos \left(2 \pi k f_{p} t+\varphi_{k}\right)
$$

where $w_{P}$ is subject's weight; $n$ is number of harmonics; and $\eta_{k}$ and $\varphi_{k}$ are the dynamic load factor (DLF) and phase angle, respectively.

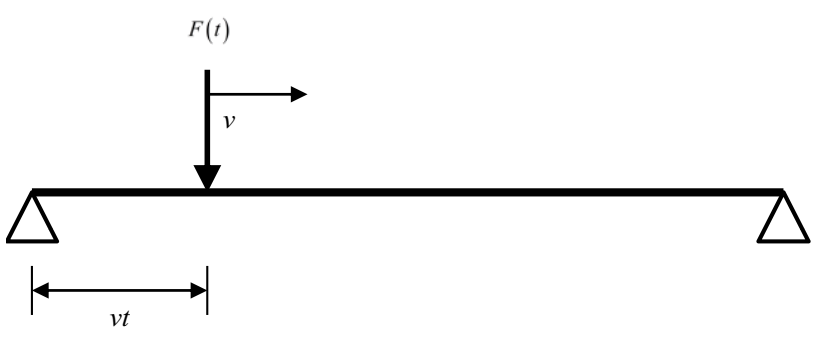

Figure 9 Analytical modelling of human-bridge system.

The system's equation of motion in modal space for mode $j$ is written as:

$$
\ddot{q}_{j}+2 \xi_{j} \omega_{j} \dot{q}_{j}+\omega_{j}^{2} q_{j}=\frac{P_{j}}{M_{j}}
$$

where $q_{j}, \dot{q}_{j}, \ddot{q}_{j}$ are the modal displacement, velocity and acceleration for mode $j$; and $\xi_{j}$ and $\omega_{j}$ are the damping and circular frequency of mode $j . P_{j}$ and $M_{j}$ are the modal force and mass for mode $j$, calculated as:

$$
P_{j}=F(t) \delta(x-v t) ; \quad M_{j}=\frac{m_{b} L}{2}
$$

in which $\delta$ is Dirac delta function; $x$ and $v$ are pedestrian location and velocity; and $m_{b}$ and $L$ are mass per unit length and length of the bridge. The modal vibration response of the bridge is obtained using Newmark- $\beta$ integration. Finally, the vibration response of the bridge in physical coordinates at any location can be calculated:

$$
\ddot{u}=\sum_{j=1}^{n} \phi_{j}(x) \ddot{q}_{j}
$$

where $\phi_{j}(x)$ is the mode shape of mode $j$ which is assumed as that of a perfectly simply-supported beam:

$$
\phi_{j}(x)=\sin \left(\frac{j \pi x}{L}\right)
$$

In this paper, only the first mode of the bridge is considered and also phase angle is taken to be zero since for each trial the subjected started from a standing start 
two paces or so back from the bridge support. Also, for comparing results with what could considered to be a state-of-the art vibration prediction approach, the first of Young's DLFs [4] which is a function of pacing frequency is used. Figure 10 shows an example of vibration response using both the recorded RS GRFs and BS GRFs at the mid-span of the bridge. From these results it is clear that HSI leads to the reduction of contact force between pedestrian and bridge.

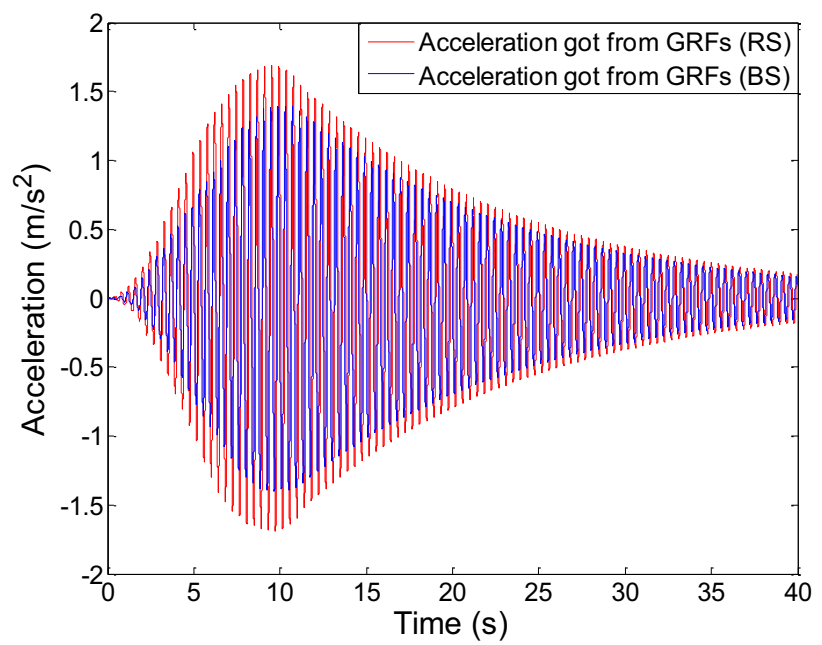

Figure 10 An example of analytical bridge vibration response due to RS and BS GRFs.

\section{Results}

As discussed earlier, the damping and frequency of the bridge for each test subject and pacing frequency is obtained from the free decay vibration response analysis. A summary of the bridge frequency and damping for each test subject and pacing frequency obtained from free vibration response is given in Table 1 and are used later. It is clear to see the influence of the test subject and pacing frequency on the bridge properties, most notably the damping.

Since frequency and damping of the bridge change during each pedestrian walking and pacing frequency, the values obtained for each test subject in Table 1 are used as bridge properties in analytical model to have a reasonable estimation of the vibration responses. The measured accelerations are filtered by low pass Butterworth filter, in which the cut-off frequency is $5 \mathrm{~Hz}$.

Figure 11 illustrates the mid-span measured acceleration and that of the analytical model subjected to BS GRFs. The experimental accelerations are found to typically be smaller than those predicted by the analytical model, even though using the measured induced force to the bridge surface. This indicates the influence of the pedestrian on the bridge properties and response which cannot be captured by the analytical model (moving force only). The difference between the analytical prediction and measured acceleration becomes more and more obvious when the response amplitude increases. This is most probably caused by an increase in the bridge damping ratio due to the presence of the walking pedestrian, and is more pronounced with increasing amplitude of vibration. The maximum value of measured acceleration commonly occurs earlier than that of the simulation value, which can be explained by the influence of the pedestrian on the damping of bridge.

Table 1. Summary of bridge frequency and damping for each test subjects obtained from free vibration decay of bridge response.

\begin{tabular}{|c|c|c|c|}
\hline \multirow{2}{*}{ TS \# } & $f_{p}(\mathrm{~Hz})$ & $\xi(\%)$ & $f_{b}(\mathrm{~Hz})$ \\
\hline \multirow{3}{*}{1} & 1.2 & 0.25 & 2.430 \\
\cline { 2 - 4 } & 2.1 & 0.3 & 2.425 \\
\cline { 2 - 4 } & 2.4 & 0.5 & 2.395 \\
\hline \multirow{3}{*}{2} & 1.2 & 0.25 & 2.420 \\
\cline { 2 - 4 } & 2.1 & 0.3 & 2.415 \\
\cline { 2 - 4 } & 2.4 & 0.5 & 2.395 \\
\hline \multirow{3}{*}{3} & 1.2 & 0.25 & 2.415 \\
\cline { 2 - 4 } & 2.1 & 0.3 & 2.410 \\
\cline { 2 - 4 } & 2.4 & 0.53 & 2.392 \\
\hline
\end{tabular}

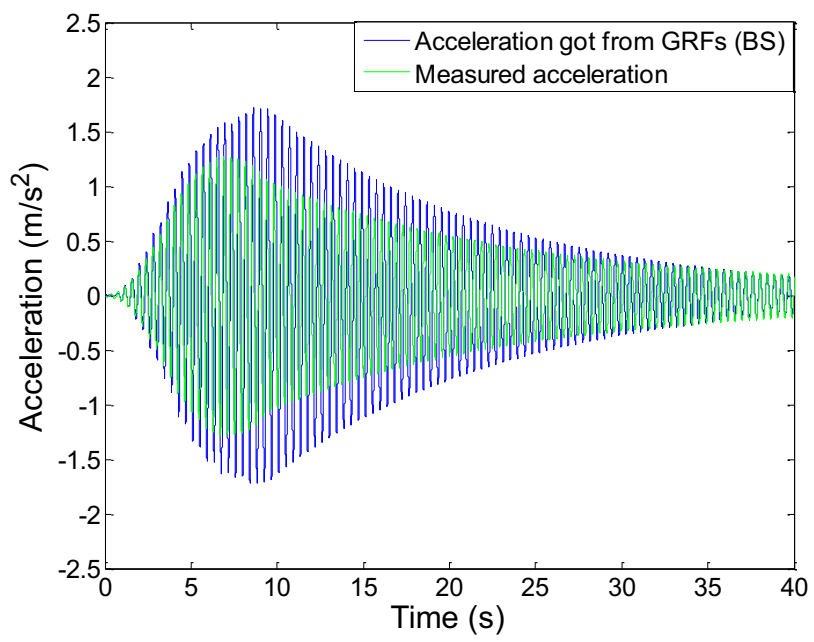

Figure 11 Measured mid-span acceleration and that of the analytical model using the BS GRFs.

Maximum mid-span accelerations $\left(a_{\max }\right)$ for measured data, simulated accelerations due to GRFs on BS, as well as the average of simulated-to-measured maxim acceleration ratio are given in Tables 2, 3, and 4 for each test subject using the bridge frequencies and damping values given in Table 1. In addition, the analytical vibration response obtained for each test subject using the DLFs of Young [4] are also given as a representative example of state-of-the-art prediction. In these tables, the average ration between the simulated and measured accelerations is given.

From the results, there is a noticeable difference between the simulated and measured values especially when the pacing frequency is $2.4 \mathrm{~Hz}$. This must be due to some change in the bridge-pedestrian system dynamic properties during the traverse, since the mode shape and mass are well-known. Also, the results using Young's 
DLFs show that while it overestimates vibration responses at pacing frequencies corresponding to $1.2 \mathrm{~Hz}$ and $2.1 \mathrm{~Hz}$, the vibration response at resonance can be underestimated.

Table 2. Maximum mid-span accelerations for test subject 1 .

\begin{tabular}{|c|c|c|c|c|}
\hline \multirow{2}{*}{$f_{p}(\mathrm{~Hz})$} & \multicolumn{3}{|c|}{$a_{\max }\left(\mathrm{m} / \mathrm{s}^{2}\right)$} & \multirow{2}{*}{$\begin{array}{c}\text { Average } \\
\text { Ratio }\end{array}$} \\
\hline & Simulated & Measured & Young & \\
\hline \multirow{4}{*}{1.2} & 0.16 & 0.17 & 0.33 & \multirow{4}{*}{0.96} \\
\hline & 0.15 & 0.15 & 0.33 & \\
\hline & 0.15 & 0.15 & 0.33 & \\
\hline & 0.14 & 0.15 & 0.32 & \\
\hline \multirow{4}{*}{2.1} & 0.16 & 0.16 & 0.12 & \multirow{4}{*}{1.03} \\
\hline & 0.21 & 0.20 & 0.12 & \\
\hline & 0.17 & 0.16 & 0.12 & \\
\hline & 0.15 & 0.15 & 0.12 & \\
\hline \multirow{4}{*}{2.4} & 1.33 & 1.11 & 1.66 & \multirow{4}{*}{1.35} \\
\hline & 1.77 & 1.25 & 1.49 & \\
\hline & 1.64 & 1.16 & 1.56 & \\
\hline & 1.56 & 1.12 & 1.61 & \\
\hline
\end{tabular}

Table 3. Maximum mid-span accelerations for test subject 2.

\begin{tabular}{|c|c|c|c|c|}
\hline \multirow{2}{*}{$f_{p}(\mathrm{~Hz})$} & \multicolumn{3}{|c|}{$a_{\max }\left(\mathrm{m} / \mathrm{s}^{2}\right)$} & \multirow{2}{*}{$\begin{array}{c}\text { Average } \\
\text { Ratio }\end{array}$} \\
\hline & Simulated & Measured & Young & \\
\hline \multirow{4}{*}{1.2} & 0.31 & 0.25 & 0.45 & \multirow{4}{*}{1.17} \\
\hline & 0.16 & 0.15 & 0.44 & \\
\hline & 0.20 & 0.20 & 0.46 & \\
\hline & 0.22 & 0.17 & 0.45 & \\
\hline \multirow{4}{*}{2.1} & 0.28 & 0.20 & 0.15 & \multirow{4}{*}{1.29} \\
\hline & 0.31 & 0.22 & 0.14 & \\
\hline & 0.27 & 0.22 & 0.14 & \\
\hline & 0.25 & 0.22 & 0.15 & \\
\hline \multirow{4}{*}{2.4} & 1.97 & 1.30 & 1.84 & \multirow{4}{*}{1.54} \\
\hline & 2.14 & 1.40 & 2.00 & \\
\hline & 2.06 & 1.31 & 2.03 & \\
\hline & 2.15 & 1.39 & 2.03 & \\
\hline
\end{tabular}

Table 4. Maximum mid-span accelerations for test subject 3 .

\begin{tabular}{|c|c|c|c|c|}
\hline \multirow{2}{*}{$f_{p}(\mathrm{~Hz})$} & \multicolumn{3}{|c|}{$a_{\max }\left(\mathrm{m} / \mathrm{s}^{2}\right)$} & \multirow{2}{*}{$\begin{array}{c}\text { Average } \\
\text { Ratio }\end{array}$} \\
\hline & Simulated & Measured & Young & \\
\hline \multirow{4}{*}{1.2} & 0.26 & 0.24 & 0.59 & \multirow{4}{*}{1.06} \\
\hline & 0.24 & 0.22 & 0.66 & \\
\hline & 0.28 & 0.24 & 0.65 & \\
\hline & 0.35 & 0.36 & 0.65 & \\
\hline \multirow{4}{*}{2.1} & 0.31 & 0.23 & 0.18 & \multirow{4}{*}{1.17} \\
\hline & 0.30 & 0.26 & 0.19 & \\
\hline & 0.28 & 0.27 & 0.19 & \\
\hline & 0.27 & 0.25 & 0.19 & \\
\hline \multirow{4}{*}{2.4} & 3.03 & 1.80 & 2.34 & \multirow{4}{*}{1.60} \\
\hline & 2.87 & 1.86 & 2.28 & \\
\hline & 2.84 & 1.84 & 2.40 & \\
\hline & 3.19 & 1.86 & 2.47 & \\
\hline
\end{tabular}

To investigate what the bridge properties may be during the traverse, following O'Sullivan et al's study [14], the damping ratios are changed such that the simulated accelerations and measured accelerations have a good match. Table 5 presents the bridge frequency and damping results for pacing frequency of $2.4 \mathrm{~Hz}$ when a good match between two types of the response is reached. It shows that bridge damping becomes 2 to 3 times the empty bridge during resonance and that there is a large change in damping. Also, the bridge frequency undergoes a slight change, most probably due to the small pedestrian-to-bridge mass ratio.

Table 5. Optimized bridge properties by matching maximum mid-span accelerations for pacing frequency of $2.4 \mathrm{~Hz}$ (resonance).

\begin{tabular}{|c|c|c|c|c|}
\hline \multirow{2}{*}{ TS \# } & \multirow{2}{*}{$\xi(\%)$} & \multirow{2}{*}{$\begin{array}{c}f_{b} \\
(\mathrm{~Hz})\end{array}$} & \multicolumn{2}{|c|}{$a_{\max }\left(\mathrm{m} / \mathrm{s}^{2}\right)$} \\
\hline & & & Simulated & Measured \\
\hline \multirow{4}{*}{1} & \multirow{4}{*}{1.2} & \multirow{4}{*}{2.395} & 1.01 & 1.10 \\
\hline & & & 1.31 & 1.25 \\
\hline & & & 1.21 & 1.16 \\
\hline & & & 1.15 & 1.12 \\
\hline \multirow{4}{*}{2} & \multirow{4}{*}{1.3} & \multirow{4}{*}{2.395} & 1.40 & 1.30 \\
\hline & & & 1.51 & 1.40 \\
\hline & & & 1.41 & 1.31 \\
\hline & & & 1.48 & 1.39 \\
\hline \multirow{4}{*}{3} & \multirow{4}{*}{1.6} & \multirow{4}{*}{2.392} & 1.95 & 1.80 \\
\hline & & & 1.85 & 1.86 \\
\hline & & & 1.82 & 1.84 \\
\hline & & & 2.03 & 1.86 \\
\hline
\end{tabular}




\section{Discussion}

The results presented above strongly indicate that humanstructure interaction is likely to be a significant source of error between the predictions of current analytical models and the measured accelerations. Interestingly, while it is sometimes found that the analytical approach using Young's DLFs gives a higher acceleration response (conservative), it can also give a lower response (nonconservative). The level of conservatism is highest for a resonant pacing frequency.

It is instructive that the comparison between the simulated response using the measured imparted force, and the actual vibration response is often significantly different, especially at resonance. Since the force is the same this points to a lack of accuracy in the analytical model. However, since the mode shape and bridge mass are well known, it then follows that it is the bridge frequency and damping that is altering during the event. Indeed, to get a better match is found that the bridge damping most particularly needs to be changed by a significant amount, doubling or even tripling.

\section{Conclusions}

In this paper, an attempt is made to quantify HSI phenomenon using a novel experimental and analytical approach. It is found that human-structure dynamic interaction is associated both with the forces that excite the structure and with the corresponding influence of humans on the dynamic properties of the structure they occupy. Most notably, the bridge damping ratio increases with the increase of pacing frequency so that apparent value of damping becomes 2 to 3 times during resonance. When properly quantified, this finding could indicate a means of accounting for human-structure interaction by incorporating rules into codes of practice that account for changing modal properties during loading events.

\section{References}

[1] S. Živanović, A. Pavic, P. Reynolds, Vibration serviceability of footbridges under humaninduced excitation: A literature review, J. Sound Vib. $279 \quad$ (2005) $1-74$. doi:10.1016/j.jsv.2004.01.019.

[2] P. Dallard, A. Flint, S. Le Bourva, A. Low, R.M.R. Smith, M. Willford, The London Millenium Footbridge, Struct. Eng. 79 (2001) 1735 .

[3] J. Blanchard, B.L. Davies, J.W. Smith, DESIGN CRITERIA AND ANALYSIS FOR DYNAMIC LOADING OF FOOTBRIDGES, in: Proceeding a Symp. Dyn. Behav. Bridg. Transp. Road Res. Lab. Crowthorne, Berkshire, Engl., 1977.

[4] P. Young, Improved floor vibration prediction methodologies. ARUP vibration seminar, (2001).
[5] M.C. Sethi, Dynamic Response of Beam Under Moving Mass,A THESIS SUBMITTED IN PARTIAL FULFILLMENT OF THE REQUIREMENTS FOR THE DEGREE OF Bachelor of Technology In Mechanical Engineering, Department of Mechanical Engineering, 2012.

[6] A. Pavic, C.H. Yu, J.M.W. Brownjohn, P. Reynolds, Verification of the existence of humaninduced horizontal forces due to vertical jumping, in: Proc. IMAC XX, Vol. 1, Los Angeles, CA, Febr. 4-7, 2002: pp. 120-126.

[7] S. Zivanovic, A. Pavic, P. Reynolds, Human structure dynamic interaction in footbridges, ICE, Bridg. Eng. 158 (2005) 165-177.

[8] M.J. Griffin, J. Erdreich, Handbook of Human Vibration, J. Acoust. Soc. Am. 90 (1991) 2213. doi:10.1121/1.401606.

[9] A.F. Cordero, Human Gait, Stumble and...fall? Mechanical limitations of the recovery from a stumble, University of Twente, Enschede, The Netherlands, 2003.

[10] A. McRobie, G. Morgenthal, J. Lasenby, M. Ringer, Section model tests on human-structure lock-in, ICE, Bridg. Eng. 156 (2003) 71-79.

[11] R. Sachse, A. Pavic, P. Reynolds, HumanStructure Dynamic Interaction in Civil Engineering Dynamics: A Literature Review, Shock Vib. Dig. 35 (2003) 3-18. doi:10.1177/0583102403035001624.

[12] S. Živanović, A. Pavić, E.T. Ingólfsson, Modeling Spatially Unrestricted Pedestrian Traffic on Footbridges, J. Struct. Eng. 136 (2010) 1296-1308. doi:10.1061/(ASCE)ST.1943$541 X .0000226$.

[13] M. Kasperski, Damping induced by pedestrians, in: 9th Int. Conf. Struct. Dyn. EURODYN,Porto, Port. 30 June - 2 July, 2014: pp. 1059-1064.

[14] D. O'Sullivan, C. Caprani, J. Keogh, The Response of a Footbridge to Pedestrians Carrying Additional Mass, in: BCRI(Bridge Concr. Res. Ireland) Conf. Dublin, 2012. 